\title{
Hot Incremental Forming of Biocomposites Developed from Linen Fibres and a Thermoplastic Matrix
}

\author{
Sandino Torres ${ }^{1, *}$ - Roberto Ortega ${ }^{1}$ - Pablo Acosta ${ }^{1}$ - Edisson Calderón ${ }^{2}$ \\ ${ }^{1}$ Central Technic Higher Technological Institute (ISTCT), Industrial Mechanics \& Electronics, Ecuador \\ ${ }^{2}$ Chimborazo Higher Polytechnic School (ESPOCH), Maintenance Research GROUP (GIMAN), Ecuador
}

The use of biodegradable materials has a growing field of application due to environmental concerns, however, scientific research on incremental forming using biomaterials is scarce. Thus, this study focuses on the single point incremental forming (SPIF) process applied to a composite sheet that combines a biodegradable thermoplastic matrix (Solanyl) reinforced with linen fibres. The influence of the process parameters on the final geometry is determined, evaluating the effect of the following factors: step depth, wall angle and temperature reached during the process. Additionally, a heated aqueous medium is incorporated which facilitates the formability of the composite sheets. This method is especially useful for materials that have poor formability at room temperature. The benefits of using controlled heat include the reduction of forming forces applied to the plate, improved accuracy due to the reduction of elastic recovery, and the manipulation of the samples remarkably close to the glass transition temperatures. Through this experimental study with the variables analysed, a maximum shaping depth of $31 \mathrm{~mm}$ is obtained. These results confirm that the single point shaping used with bioplastic materials is possible and has positive outcomes for incremental forming.

Keywords: incremental forming, bio-composites, hot formability, analysis of variance, deformation

Highlights

- The factors that contribute to improve the hot incremental forming of composites with a thermoplastic matrix and their efficacy are identified.

- This article gathers the experimental results of the single point forming process in sheets of composite material (Solanyl-linen fibres).

- The main factors that influence the hot incremental forming of biodegradable composite materials are established.

- The methodology used is validated due to the small differences that exist between the different results, showing the significance of each of the factors analysed with the part depth reached during the conformation process.

\section{INTRODUCTION}

Material processing methods are being continuously modified due to technological development and the need to process materials faster. One method of processing materials, called incremental forming, is a technique that progressively deforms a sheet of material, using a tool operated by numerical control machines. The trajectory of the tool is generated with a computer aided manufacturing (CAM) software from a three-dimensional model that reproduces the desired geometry. Initially, the single point incremental forming (SPIF) process was applied to metallic materials for small batch production. This technique is focused on creating prototypes of materials; however, the complex methods of conventional tooling for forming processes represent a limitation due to the high investment for the manufacturing process.

Currently, there are several practical applications of incremental forming in the automotive and aeronautical sector. In Japan, for example, incremental forming is used as an alternative process to add certain features to previously stamped vehicle panels [1]. The fields of application also include the medical sector, where several studies have been carried out for the implementation of this process in the manufacture of a femoral component of titanium-tantalum [2]. In addition, the design and manufacturing process has been analysed for using the same technique in a cranial prosthesis [3], obtaining quite encouraging results. Also, the performance of the forming process is important from the perspective of geometric precision. The manufacture of biomedical implants, through the incremental forming process, has reached deviations in the range of $1 \mathrm{~mm}$ to $2 \mathrm{~mm}$, using the variation of the tool path as a method to improve the dimensional accuracy of the final product [4]

Recently, innovative proposals have been made to the incremental forming process, especially to improve the formability. One of the variations utilised is the application of heat, where incremental forming processes have been carried out by means of localised heating, achieving a high degree of deformation [5]. The heat-assisted incremental sheet forming (HAISF) has been applied to titanium alloys and high strength steel, reaching $600{ }^{\circ} \mathrm{C}$ with localized heat that comes from methods such as: laser heat, friction heat, electrical heat and induction heating [6]. In addition, 
there are studies of incremental shaping applied to stainless steel dental prostheses with a plate thickness of $0.5 \mathrm{~mm}$, allowing to improve the production of custom-made components [7]. The initial tests, where higher temperatures were assigned to the areas to be shaped, showed the viability of this technique [8]. There are different techniques for the application of heat, one of which is to use fluid as a heating medium to form magnesium parts. In this case, reduced forces were required for formation at elevated temperatures and improved formability was achieved [9]. It has also been determined that incremental forming with hot air is a suitable method for parts production of prostheses and orthoses, which are implemented in medical applications [10].

Moreover, the incremental forming of polymers with materials such as polyoxymethylene (POM), polytene (PE), polyamide (PA), polyvinyl chloride (PVC), polycarbonate (PC) and its manufacture of complex components has been investigated with the application of heat [11]. Studies show that with polystyrene sheets, where hot air is applied using a nozzle to raise the temperature towards the forming area, the formability is improved [9]. Temperature plays a particularly important role in the behaviour of plastic materials due to their glass transition, fluidity, morphology, and degradation characteristics. In addition, studies of incremental forming in composite materials and their preliminary viability have been carried out using glass fibre reinforced polyamide [12], which is a widely used material in the automotive sector.

Clear identification of strain conditions during the incremental forming process defines the degree of formability of a thermoplastic material. The evolution of the process shows stretching conditions and through-thickness shear deformation. Unfortunately, the physical mechanism of deformation in thermoplastics subjected to incremental shaping is not yet fully understood [13]. In the field of nanocomposites with a thermoplastic matrix, the addition of alloying materials directly influences the characteristics of the resulting composite material. PA 12 combined with $1 \%$ to $3 \%$ Cloisite 93A, for example, improves the mechanical strength of the composite material by $4 \%$ to $6 \%$ [14].

One of the other main variables of the incremental forming process is the spindle speed. However, the spindle can consume a large amount of energy. It has been said that the energy consumption will reduce if the tool does not rotate, and if the machining repetitions are decreased [15]. The spindle speed is important as it has the ability to reduce the maximum forming forces needed and increase the formability of the sheet [16].

This study analyses the effects of various parameters (in-depth tool feed and force) on the forming behaviour of a biodegradable thermoplastic matrix (manufacture name: Solanyl), which has been combined with short linen fibres, with a length of no more than $3 \mathrm{~mm}$, and linen fibres reinforcement with a concentration of $10 \%$. The granulated material is transformed into plates of material of $3 \mathrm{~mm}$ thickness and size of $180 \mathrm{~mm} \times 150 \mathrm{~mm}$ by means of the injection process: The plasticizing unit of the injection machine is in charge of heating the material to a temperature of $150{ }^{\circ} \mathrm{C}$ and introducing it into the mold that reproduces the geometry of the plates. The plates are used in the incremental forming process, using a fluid heat source to improve its formation. Polymers reinforced with natural fibres can be used to make lightweight constructions due to their increased strength [17].

The challenge is to present the main forming parameters for prototype production. Therefore, the present study focuses mainly on the evaluation of the maximum forming depth under the influence of a heat source. For this reason, a hot environment is used through a fluid (water), at a temperature based on the obtained working range of the material. In this way, it is determined how the material behaves with the different process parameters, both constant and variables such as feed rate, tool diameter, material elasticity, temperature, spindle speed and step depth.

\section{METHODS}

The experimental methodology is based on simultaneous sampling of force and temperature applied to the material to analyse the deformation in a sheet of thermoplastic material of natural origin, and in a sheet of composite material, during the incremental forming process. The force $[\mathrm{N}]$ that the tool exerts on the material and the temperature applied to the sheet is recorded. The conical geometry formed is shown in Fig. 1; where $\varnothing D$ is the largest diameter of the cone, $\beta$ is the angle of inclination, and $h$ is the total length reached in the forming process. This is the geometry profile used for the tool trajectory, taking into account that the tool diameter will cause the formation of tracks in the corners of the model.

A spherical-tipped tool was selected, which creates a descending circular path to form the desired geometry. The forming process is carried out inside of an aqueous medium, using an electric heater to increase the water temperature; consequently, the 
testing sheets are completely submerged into the fluid. The temperature is controlled through an electronic circuit that maintains the selected temperature ranges. The presence of the aqueous heating medium guarantees the heat transfer by convection in a homogeneous way towards the composite sheet. The fluid temperature is monitored by a thermocouple, and the data is collected in real time through a remotecontrol system. Different parameters were selected that intervene in the incremental forming process, and through this work the impacts caused by these variables and their interactions in the forming of this type of material are studied.

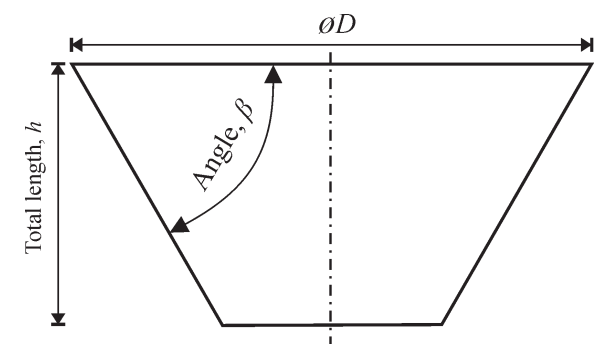

Fig. 1. Geometry profile for the incremental forming process

\section{EXPERIMENTAL}

This experiment aims to determine the influence of the different parameters involved in the incremental forming process, and to understand their interactive effects. These experiments focus on different forming parameters based on previous research [12] and [16]. These previous investigations show the main factors that influence the viability of the incremental forming process and provides a general criterion regarding the results. For this reason, the following parameters are evaluated: wall angle $(\beta)$, step depth and temperature. For best results, the spindle is disconnected from the machine in such a way as to limit the effects of friction and avoid possible bending or twisting defects. Additionally, the maximum speed of the machine is established using a tool with a $6 \mathrm{~mm}$ diameter, to analyse the influence of these parameters on the total depth.

Table 1 shows a summary of the variables and parameters involved in the process.

In this study, plates injected with a starch-based thermoplastic (Solanyl C1201 from Rodenburg Biopolymers) or a composite material formed by this thermoplastic matrix with $10 \%$ of its weight in linen fibres, were used. The linen fibre is cut with a length less than $3 \mathrm{~mm}$, to improve the processing conditions of the composite material.
Table 1. Parameters involved in the incremental forming process

\begin{tabular}{|c|c|c|}
\hline Parameters & \multicolumn{2}{|c|}{ Constant } \\
\hline Tool diameter [mm] & \multicolumn{2}{|c|}{6} \\
\hline Feed rate $[\mathrm{mm} / \mathrm{min}]$ & \multicolumn{2}{|c|}{500} \\
\hline Spindle speed(rpm) & \multicolumn{2}{|c|}{0} \\
\hline Parameters & \multicolumn{2}{|c|}{ Variables } \\
\hline Step depth [mm] & 0.2 & 0.4 \\
\hline Wall angle $\left[{ }^{\circ}\right]$ & 45 & 60 \\
\hline Temperature $\left[{ }^{\circ} \mathrm{C}\right]$ & 80 & 90 \\
\hline
\end{tabular}

Previously, tensile tests are carried out to analyse the mechanical behaviour of these materials [18].

The results of the mechanical properties of these materials are presented in Table 2.

Table 2. Results of the mechanical properties of the materials used

\begin{tabular}{cccc}
\hline Material & $\begin{array}{c}\text { Ultimate tensile } \\
\text { strength [MPa] }\end{array}$ & $\begin{array}{c}\text { Elastic } \\
\text { modulus [-] }\end{array}$ & $\begin{array}{c}\text { Percentage of } \\
\text { deformation at } \\
\text { break [\%] }\end{array}$ \\
\hline Biopolymer & 10.14 & 2857.25 & 0.34 \\
\hline $\begin{array}{c}\text { Biopolymer }+ \\
10 \% \text { linen fibre }\end{array}$ & 10.16 & 3075.54 & 2.15 \\
\hline
\end{tabular}

Through these results, the behaviour of these materials and the influence of its properties on the incremental forming process are analysed. For this reason, the elastic modulus is used as a reference to analyse its interaction with other parameters of the forming process, quantifying the improvement in formability.

The manufacturing process of this compound alternates the extrusion and injection process, as presented in [23]. The tooling and tooling design are made according to similar configurations shown in other studies of incremental shaping [3]. In this case, the main apparatus incorporates the fluid (water) at different controlled temperatures. Fig. 3 shows the apparatus used in the study.

The material used is a thermoplastic, for this reason a thermal characterization technique called differential scanning calorimetry (DSC) is carried out. DSC is a dynamic procedure that incorporates a thermo analysis technique, which determines the amount of heat that a substance absorbs or releases when it is subjected to a variation of heat flow. Thanks to this procedure, it is possible to establish at what temperature physical or chemical changes occur, including boiling points, crystallization, glass transitions, hydration effect, phase changes, curing, among others. This procedure simplifies the analysis of any type of polymer material, such as cement, ceramics, metals, pure organic matter, 
pharmaceuticals, liquid crystals, biological systems, electrical and metallurgical industry [19] to [23].

Fig. 2 shows a change or energy jump that indicates a change in the properties of the material, so for example at $150{ }^{\circ} \mathrm{C}$ the curve falls into a sink that, under references of similar materials, indicates the process of fusion. But at exactly $82{ }^{\circ} \mathrm{C}$ the last decline in the graph can be seen, which suggests the glass transition temperature, the temperature necessary to be able to form the material, without losing its viscoelastic properties. This test was performed with an instrument DSC Q2000 V24.9 (origin USA) using the Sapphire method. The characterization of the material makes it possible to determine the glass transition and melting temperatures; The first is important to carry out the plastic deformation of the composite material; the second, intervenes directly in the shaping of the material. Consequently, the test is carried out to determine the thermal parameters of the polymeric material.

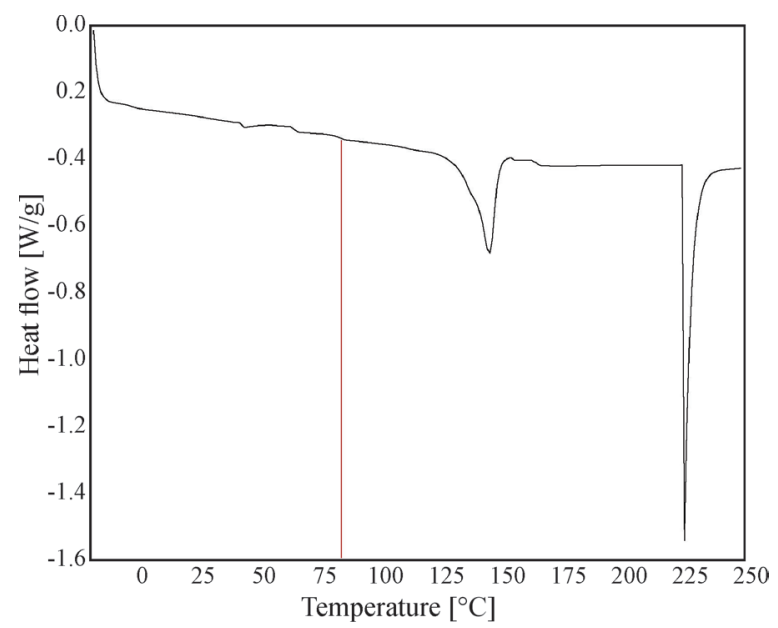

Fig. 2. DSC thermal test results for the sheet of thermoplastic, trade name Solanyl

\subsection{Experimental Equipment}

The tool is built using AISI 4340 alloy steel with a spherical tip and $6 \mathrm{~mm}$ diameter shank, for the forming process; additionally, a SIEG CNC (China), milling machine is used. The machining program is carried out in the Autodesk HSM software, where toolpaths are generated, with Z-level strategy. The steps are made in the direction of the $\mathrm{Z}$-axis, the information is post processed and the machining program is generated in $\mathrm{G}$ code, for a FANUC controller.

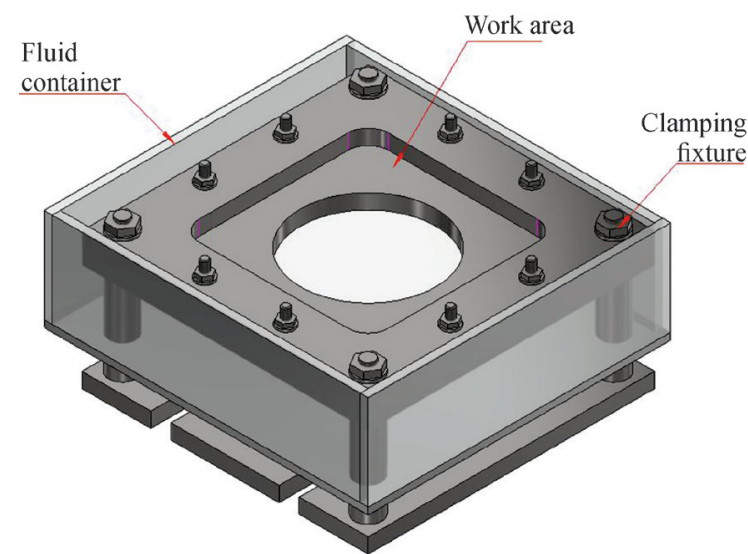

Fig. 3. Fluid container used for the hot incremental forming process

The characterization of the biodegradable materials used in this study, allowed us to understand the physical, chemical, and mechanical properties, in particular, the temperature parameters that are needed to process the material [24], where the elastic modulus is also selected as a reference in order to evaluate the results of the interaction of this parameter with the temperature, wall angle, and step down variables. In Figure 4, below, the components for the incremental forming process in biodegradable plates are shown, for which a source of heat is applied within an aqueous environment.

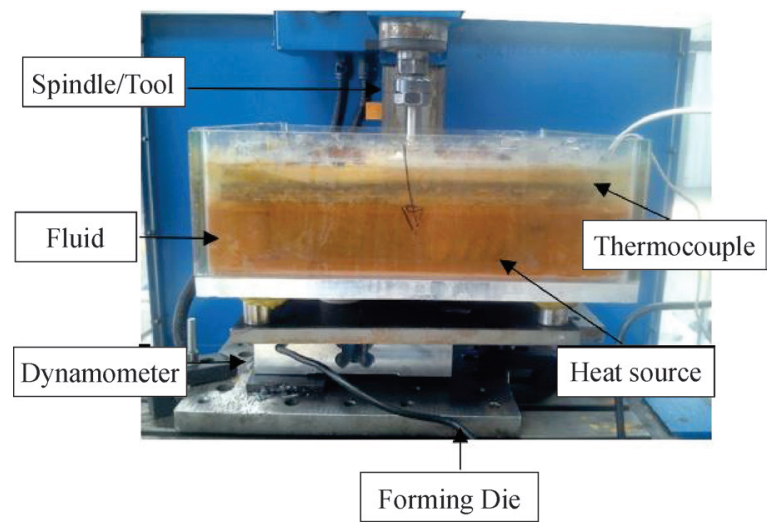

Fig. 4. Experimental configuration for hot incremental forming process

\subsection{Electronic Device}

To obtain and transmit data, an electronic circuit consisting of sensors, actuators and a remote-control system that displays data in real time through a userfriendly, human-machine interface has been designed and implemented. For data collection, a $\mathrm{K}$ type 
thermocouple is placed inside the fluid area, which measures the temperature in a range of $0{ }^{\circ} \mathrm{C}$ to $800{ }^{\circ} \mathrm{C}$, as well as a weight sensor with a capacity of $40 \mathrm{kN}$, which is responsible for recording the force generated during the process between the machine table and the forming die, which weighs the whole system, and this data is then transformed into a force value. The sensors are controlled by an electronic "Arduino Uno" card, and the programming is done in C language, using the Arduino APK environment. Temperature and weight data are obtained by direct connection to the Arduino card; this data is sent to a local server, a Raspberry Pi 3 card programmed in Python. Fig. 5 shows the data collection diagram.

The information collected is sent via ethernet or wi-fi to a remote client, in this case a personal computer (PC), which receives the data through a program carried out in a virtual instrumentation software: Labview, where the value of temperature is received and shown in degrees Celsius $\left[{ }^{\circ} \mathrm{C}\right]$ and weight is transformed into force and shown in Newtons $[\mathrm{N}]$. The LabView program is used for data collection (Fig. 6).

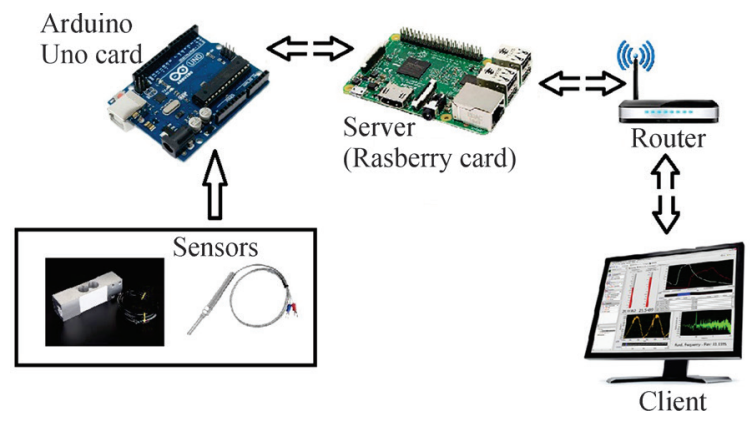

Fig. 5. Data collection diagram

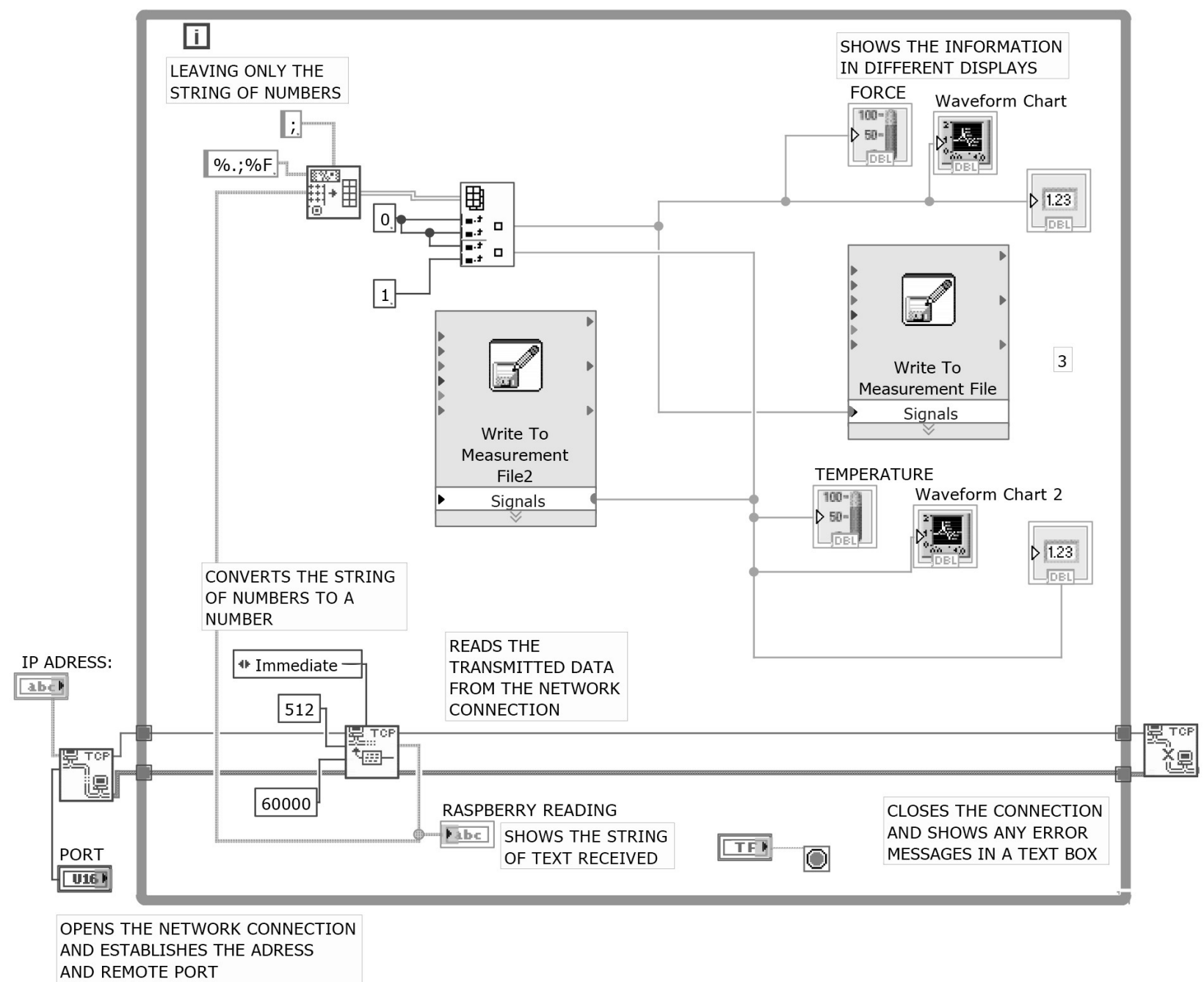

Fig. 6. Data collection diagram 


\section{RESULTS}

The experiments are carried out to determine the most influential factors on the total part depth with the incremental forming process. For this, the parameters of step depth, wall angle and temperature of the aqueous medium are manipulated. In addition, the behaviour of the force in the incremental forming process is studied. The results obtained from the depth are taken directly from the CNC machine controller, once the material breaks. The depth value is recorded once it fails, that is, when a crack or fracture occurs in the sheet. In the event that the test meets the total depth of the proposed geometry, the length obtained is measured and that value as the maximum depth reached.

To obtain precise results, a $2^{4}$ factorial design with three replicas is made in Statgraphics. The results are evaluated by analysing the variance (ANOVA), which establishes the level of relationship between the variables. The response variable constitutes the total length reached after the incremental forming process. The factors for the ANOVA are presented in Table 3.

Table 3. Factors for analysis of variance

\begin{tabular}{lccc}
\hline Factors & Low $(-1)$ & High (1) & Continuous \\
\hline A: Temperature & $80^{\circ} \mathrm{C}$ & $90^{\circ} \mathrm{C}$ & Yes \\
\hline B: Wall angle & $45^{\circ}$ & $60^{\circ}$ & Yes \\
\hline C: Step down & $0.2 \mathrm{~mm}$ & $0.4 \mathrm{~mm}$ & Yes \\
\hline D: Elastic modulus & $2857 \mathrm{MPa}$ & $3076 \mathrm{MPa}$ & Yes \\
\hline
\end{tabular}
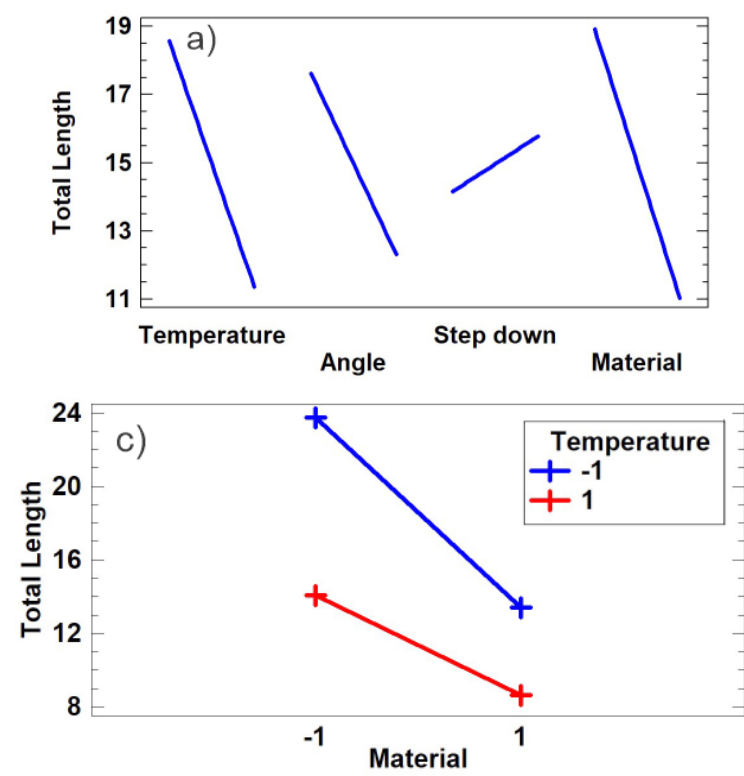

The purpose of carrying out the tests, shown in Table 3 , is to analyse the impact caused by each of these interactions with the response variable. This procedure performs a multifactor analysis of variance for in-depth feed (pitch) of the tool. Several tests and graphs are constructed to determine which factors have a statistically significant effect on the response variable. This study also provides sufficient data to test for significant interactions between the factors. For statistical analysis, a significance level of 0.05 is used with a confidence interval of $95 \%$.

Table 4. Tests performed for analysis of variance

\begin{tabular}{lcccc}
\hline Run & $A\left[{ }^{\circ} \mathrm{C}\right]$ & $\left.B{ }^{\circ}\right]$ & $C[\mathrm{~mm}]$ & $D[\mathrm{MPa}]$ \\
\hline 1 & - & - & - & + \\
\hline 2 & - & + & - & - \\
\hline 3 & - & - & - & - \\
\hline 4 & + & + & + & - \\
\hline 5 & + & - & + & - \\
\hline 6 & + & + & - & + \\
\hline 7 & + & - & - & - \\
\hline 8 & + & + & + & + \\
\hline 9 & + & - & + & + \\
\hline 10 & - & + & + & + \\
\hline 11 & + & - & - & - \\
\hline 12 & - & - & + & - \\
\hline 13 & - & + & + & - \\
\hline 14 & + & + & - & + \\
\hline 15 & - & - & + & + \\
\hline 16 & - & + & - & + \\
\hline
\end{tabular}
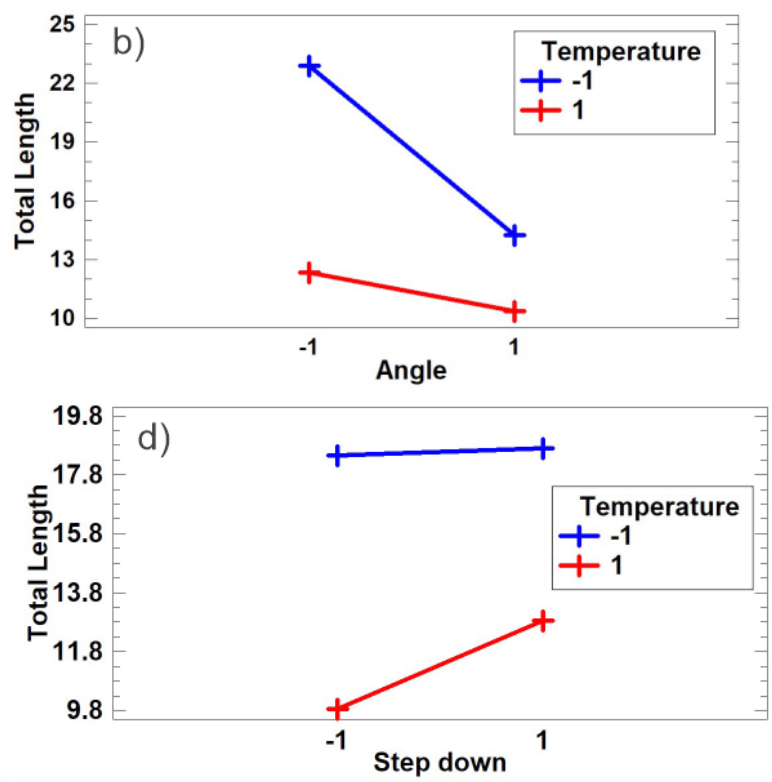

Fig. 7. Variable interaction graphics; a) main effects plot for total length, b) interacion plot for total length and angle, c) interacion plot for total length and material, d) interacion plot for total length and step down 
The results of the model obtained through the ANOVA are shown in Table 4, where the level of significance of each factor is established. Fig. 7a presents the effects of the factors with respect to the response variable and their impact on the total part length. The Figs. 7b, $\mathrm{c}$ and d show the interaction of two factors with the experimental factor; if two factors do not interact with each other, the lines on the graph are almost parallel.

The ANOVA table (Table 5) shows the different factors such as the wall angle, temperature of the heating chamber, elastic modulus of the materials, step down, and its effect on the response variable (depth), as well as the interaction with the different factors. The $P$ values prove the statistical significance of each of the factors, $P<0.05$ values have a significant effect on the response variable (total length) with a 95 $\%$ confidence level. Table 5 also shows the value for $R^{2}(94.66 \%)$, which indicates the variability related to the response variable (total lenght). The significant values are shown in Fig. 7, which relate all the factors. The results indicate that the use of temperatures of 80 ${ }^{\circ} \mathrm{C}(-1)$, angles of inclination of the geometry of $45^{\circ}$ $(-1)$ and the thermoplastic material in a natural state have statistical significance, demonstrating that for incremental forming processes the results are not as encouraging with the use of fragile materials.

Table 5. Data obtained through ANOVA analysis

\begin{tabular}{lccccc}
\hline Source & $\begin{array}{c}\text { Sum of } \\
\text { Squares }\end{array}$ & Df & $\begin{array}{c}\text { Mean } \\
\text { Square }\end{array}$ & $F$-Ratio & $P$-Value \\
\hline$A$ & 624.963 & 1 & 624.963 & 195.41 & 0.0000 \\
\hline$B$ & 338.141 & 1 & 338.141 & 105.73 & 0.0000 \\
\hline$C$ & 31.3633 & 1 & 31.3633 & 9.81 & 0.0035 \\
\hline$D$ & 745.763 & 1 & 745.763 & 233.19 & 0.0000 \\
\hline$A B$ & 132.668 & 1 & 132.668 & 41.48 & 0.0000 \\
\hline$A C$ & 22.9633 & 1 & 22.9633 & 7.18 & 0.0112 \\
\hline$A D$ & 74.0033 & 1 & 74.0033 & 23.14 & 0.0000 \\
\hline$B C$ & 0.44083 & 1 & 0.44083 & 0.14 & 0.7127 \\
\hline$B D$ & 13.4408 & 1 & 13.4408 & 4.20 & 0.0479 \\
\hline$C D$ & 085.333 & 1 & 0.85333 & 0.27 & 0.6087 \\
\hline$T$ error & 111.935 & 35 & 3.19814 & 1.954 & \\
\hline$T$ (corr.) & 2097.39 & 47 & & & \\
\hline$R$-squared & 94.6631 per & 1 & & & \\
\hline
\end{tabular}

It is evident that some factors are significant in the case of forming, as shown in the results. The result of this analysis shows that not all the parameters influence the final result, however, it is possible to determine how the different variables interact in order to maximize the result, that is, to obtain the maximum depth of the sheet. Furthermore, it is shown that bidirectional interactions are also relevant within the process.

Incremental forming test with heat source in Solanyl thermoplastic material sheet, achieving 31 $\mathrm{mm}$ in depth, as shown in Fig. 8.

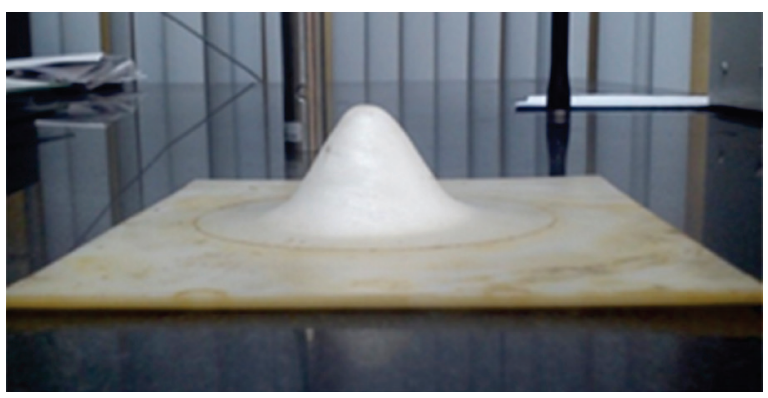

Fig. 8. Incremental forming test with heat source in Solanyl thermoplastic material sheet

The maximum step depth reached is $31 \mathrm{~mm}$ with a force of $35.25 \mathrm{~N}$ for the Solanyl material without any fibrous reinforcement. On the other hand, the lowest formability achieved is with values of total part length of $4.6 \mathrm{~mm}$ and with a force of $172.74 \mathrm{~N}$. The combination of the parameters of wall angle $(\beta)$, step down $(F z)$, and temperature $(T)$ is described in Table 6.

Table 6. Formability parameters for unreinforced Solanyl and $10 \%$ reinforced Solanyl

\begin{tabular}{lccc}
\hline Material & $\beta\left[^{\circ}\right]$ & $F z[\mathrm{~mm}]$ & $T\left[^{\circ} \mathrm{C}\right]$ \\
\hline Solanyl & 45 & 0.4 & 80 \\
\hline Solanyl $(10 \%)$ & 60 & 0.2 & 90 \\
\hline
\end{tabular}

The individual effects of the temperature, wall angle, step depth and elastic modulus factors on each material in the case of the maximum forming depth do not result in a significant effect on the response variable. However, the effect on the depth of each cited factor can be differentiated. Fig. 9 shows the contribution of each of the factors with the predominance of temperature for shaping the material as proposed in this work.

High strength sheet metal materials can barely be formed by conventional single-point incremental forming process [25]. The formed pieces produced using this process, exhibit diminished geometric precision and low forming values; thus, the application of temperature is convenient. 


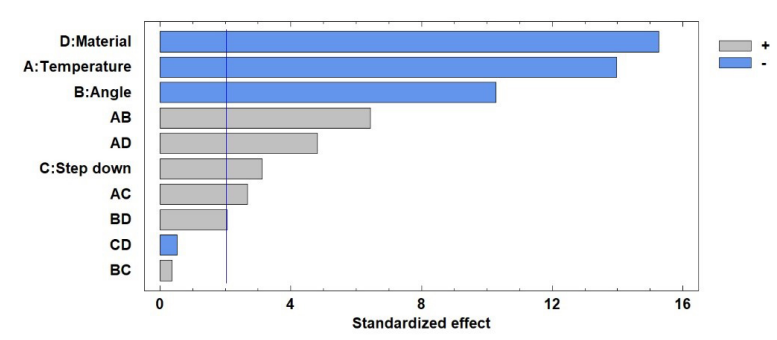

Fig. 9. Pareto diagram for contributing factors in the case of depth

In the same way, in polymer forming it has been shown that the increase in temperature will improve ductility [26], which contributes to the forming of thermoplastic materials. In this study, a homogeneous temperature was applied on the biodegradable material plate, obtaining a uniform distribution of stress and heat in the process. Unlike cold plastic deformation [9], with controlled temperatures, bio-polymeric plates can result in large deformations when analysing the displaced depth.

\section{DISCUSSION}

The results show that the thermoplastic matrix in its original state has the largest formability, because the material reaches a greater step depth compared to the composite sheet. The depth achieved in the composite material is fully congruent with the formability expected in less ductile materials (higher elastic modulus); this, without limiting the factors analysed that have statistical significance.

The results presented are summarized to provide an overview of the interaction between the different process parameters and their variables. This information presents an initial model for the forming of composite materials with a thermoplastic matrix. The results highlight factors that contribute to the forming of these types of materials and their efficacy are identified, as in the case of the forming force. Shown in Fig. 10, the forces obtained in the incremental forming process using the parameters that reach maximum forming depth, and the factors with minimum forming depth are shown in Tables 5 and 6 respectively. It is remarkable that a small increase in the temperature value of the sheet can cause some modifications in the molecular structure of the polymer. In the case of the thermoplastic matrix (Solanyl), the temperature of the glass transition shown in the DSC tests is $82{ }^{\circ} \mathrm{C}$, when this value increases, the results of the forming decrease. The temperatures applied $\left(80^{\circ} \mathrm{C}, 90^{\circ} \mathrm{C}\right)$ during the tests take the glass transition temperature as a reference and were reached after an iterative process with the variables involved not affecting the integrity of the biomaterial and composite material plates.

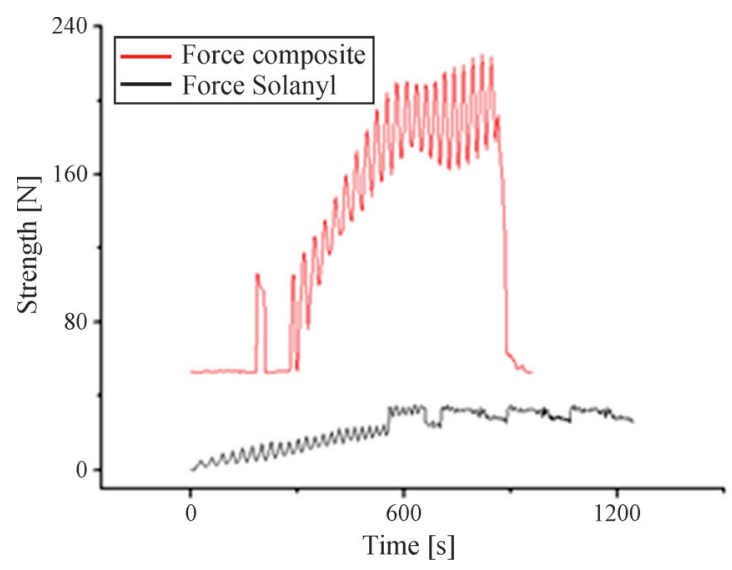

Fig. 10. Relation of forces for parameters of the maximum and minimum formability

This study shows that the improvement of the forming in thermoplastic sheets depends on the variation of certain parameters such as temperature, the angle of inclination of the geometry, and the elastic modulus of the material to be shaped.

\section{CONCLUSIONS}

This article gathers the experimental results of the single point forming process in sheets of Solanyl and composite material (Solanyl-linen fibres). According to the multifactor analysis of the variance, it is observed that the temperature of the aqueous medium has a significant effect on the forming process of sheets of the composite material with greater depths achieved at a temperature of $80^{\circ} \mathrm{C}$.

Additionally, the elastic modulus directly influences the deformation of the composite material, and therefore it is necessary to use less fragile and more ductile composite materials. The main factors that influence the forming of biodegradable composite materials are established, for which it would be important to develop a composite material with better forming properties, since this technique could achieve good results in the manufacture of products with these materials.

The methodology used is validated due to the small differences that exist between the different results, showing the significance of each of the factors analysed with the depth reached during the conformation process. The heating method used (plates in an aqueous medium) guarantees uniform temperature distribution throughout the thickness of 
the plate; however, future research must be carried out to determine the impact of hygroscopicity on polymer properties during immersion.

\section{ACKNOWLEDGEMENTS}

This study has been developed with the grant and subsidy of the project called: "Combined process of incremental forming and injection molding in sheets of biodegradable thermoplastic material", where the following academic and administrative public institutions from Ecuador are involved: "Secretaría de Educación Superior, Ciencia, Tecnología e Innovación" (SENESCYT), "Instituto Tecnológico Superior Central Técnico (ISTCT)" and "Escuela Politécnica Nacional (EPN)".

\section{REFERENCES}

[1] Amino, M., Mizoguchi, M., Terauchi, Y., Maki, T. (2014). Current status of 'Dieless' Amino's incremental forming. Procedia Engineering, vol. 81, p. 54-62, D0l:10.1016/j. proeng.2014.09.128.

[2] Bhoyar, P.K., Borade, A.B. (2015). The use of single point incremental forming for customized implants of unicondylar knee arthroplasty: A review. Research on Biomedical Engineering, vol. 31, no. 4, p. 352-357, Dol:10.1590/24464740.0705 .

[3] Centeno, G., Morales-Palma, D., Gonzalez-Perez-Somarriba, B., Bagudanch, I., Egea-Guerrero, J.J., Gonzalez-Perez, L.M., García-Romeu, M-L., Vallellano, C. (2017). A functional methodology on the manufacturing of customized polymeric cranial prostheses from CAT using SPIF. Rapid Prototyping Journal, vol. 23, no. 4, p. 771-780, Dol:10.1108/RPJ-022016-0031.

[4] Cheng, Z., Li, Y., Xu, C., Liu, Y., Ghafoor, S., Li, F. (2020). Incremental sheet forming towards biomedical implants: a review. Journal of Materials Research and Technology, vol. 9, no. 4, p. 7225-7251, D0l:10.1016/j.jmrt.2020.04.096.

[5] Al-Obaidi, A., Kräusel, V., Landgrebe, D. (2016). Hot singlepoint incremental forming assisted by induction heating. The International Journal of Advanced Manufacturing Technology, vol. 82, no. 5-8, p. 1163-1171, Dol:10.1007/s00170-0157439-x.

[6] Liu, Z. (2018). Heat-assisted incremental sheet forming: a state-of-the-art review. The International Journal of Advanced Manufacturing Technology, vol. 98, no. 9-12, p. 2987-3003, D0l:10.1007/s00170-018-2470-3.

[7] Milutinović, M., Lendjel, R., Baloš, S., Zlatanović, D.L., Sevšek, L., Pepelnjak, T. (2021). Characterisation of geometrical and physical properties of a stainless steel denture framework manufactured by single-point incremental forming. Journal of Materials Research and Technology, vol. 10, p. 605-623, DOI:10.1016/j.jmrt.2020.12.014.

[8] Gagliardi, F., Ambrogio, G., Filice, L. (2017). Incremental forming with local induction heating on materials with magnetic and non-magnetic properties. Procedia Engineering, vol. 183, p. 143-148, Dol:10.1016/J.proeng.2017.04.037.

[9] AL-Obaidi, A., Graf, A., Kräusel, V., Trautmann, M. (2019). Heat supported single point incremental forming of hybrid laminates for orthopedic applications. Procedia Manufacturing, vol. 29, p. 21-27, DOI:10.1016/j.promfg.2019.02.101.

[10] Galdos, L., De Argandoña, E.S., Ulacia, I., Arruebarrena, G. (2012). Warm incremental forming of magnesium alloys using hot fluid as heating media. Key Engineering Materials, vol. 504-506, p. 815-820, D0l:10.4028/www.scientific.net/ KEM.504-506.815.

[11] Centeno, G., Silva, M.B., Cristino, V.A.M., Vallellano, C., Martins, P.A.F. (2012). Hole-flanging by incremental sheet forming. International Journal of Machine Tools and Manufacture, vol. 59, p. 46-54, D0I:10.1016/j.ijmachtools.2012.03.007.

[12] Conte, R., Ambrogio, G., Pulice, D., Gagliardi, F., Filice, L. Incremental Sheet forming of a composite made of thermoplastic matrix and glass-fiber reinforcement. Procedia Engineering, vol. 207, p. 819-824, D0l:10.1016/j. proeng.2017.10.835.

[13] Zhu, H., Ou, H., Popov, (2020). Incremental sheet forming of thermoplastics: a review. The International Journal of Advanced Manufacturing Technology, p. 565-587, DOI:10.1007/s00170-020-06056-5.

[14] Borić, A., Kalendová, A., Urbanek, M., Pepelnjak, T. (2019). Characterisation of polyamide (PA) 12 nanocomposites with montmorillonite (MMT) filler clay used for the incremental forming of sheets. Polymers, vol. 11, no. 8, art. ID 1248, DOl:10.3390/polym11081248.

[15] Bagudanch, I., Garcia-Romeu, M.L., Sabater, M. (2016). Incremental forming of polymers: Process parameters selection from the perspective of electric energy consumption and cost. Journal of Cleaner Production, vol. 112, p. 10131024, Dol:10.1016/j.jclepro.2015.08.087.

[16] Bagudanch, I., Garcia-Romeu, M.L., Centeno, G., Elías-Zúñiga, A., Ciurana, J. (2015). Forming force and temperature effects on single point incremental forming of polyvinylchloride. Journal of Materials Processing Technology, vol. 219, p. 221229, D0I:10.1016/J.jmatprotec.2014.12.004.

[17] Sanal, l., Verma, D. (2019). Construction materials reinforced with natural products. Handbook of Ecomaterials, vol. 3, p. 2119-2142, DOI:10.1007/978-3-319-68255-6_75.

[18] Calderón Freire, E.F., TorresGallegos, L., Ortega Espín, A. (2019). Manufacture of biodegradable material based on thermoplastic polymerscombined with short flax fibers FIGEMPA Research And Development, vol. 1, no. 1, p. 32-38, Dol:10.29166/revfig.v1i1.1402. (in Spanish)

[19] Venegas Vásconez, D. (2017). Calorimetria diferencial de barrido, Termodinámica, University of the Bío Bío, Concepción, p. 1-6, DOI:10.13140/RG.2.2.12849.79204. (in Spanish)

[20] Romero Carrión, V., Tirado Rengifo, A., Dávalos Prado, J. (2015). Experimental determination using DSC of the thermal stabilities and the calorific capacities: quinua, kiwicha and cañihua. Cátedra Villarreal, vol. 1, no. 2, p. 47-52, DOI:10.24039/cv20153144. (in Spanish)

[21] Granados Cristancho, Y.A. (2015). Proyecto presentado como prerrequisito para optar al título de Ingeniero Topográfico / 
Project presented as a prerequisite to qualify for the title of Topographic Engineer.

[22] Ma, S., Yu, J.Y. (2017). Transition-based versus statebased reward functions for MDPs with Value-At-Risk. 55th Annual Allerton Conference on Communication, Control, and Computing, p. 974-981, D0l:10.1109/ ALLERTON.2017.8262843.

[23] Xie, X.Q., Moring, J., Makriyanni, A. (1997). Differential scanning calorimetry and small angle $X$-ray diffraction study of the interaction of (R)-PAF, (R)-ET-18-OMe and (R)-Lyso-PAF with model membranes. Life Sciences, vol. 61, no. 9, p. 909-923, DOI:10.1016/S0024-3205(97)00593-6.
[24] Torres Gallegos, L. (2019). Characterization of biodegradable materials for incremental forming. $5^{\text {th }}$ International Congress of Science, Technology and Innovation for Society, p. 290299.

[25] Silva, M.B., Skjoedt, M., Martins, P.A.F., Bay, N. (2014). Single Point incremental forming of metal sheets. Annual Winter Meeting of Danish Society for Metallurgy, p. MBS1-MBS14.

[26] Hughes, S.E. (2009). Materials and Their Weldability. A Quick Guide to Welding and Weld Inspection, ASME Press, p. 36-48, DOI:https://doi.org/10.1115/1.859506.ch4. 